QUARTERLY OF APPLIED MATHEMATICS

VOLUME LXIII, NUMBER 1

MARCH 2005, PAGES 56-70

S $0033-569 X(05) 00951-3$

Article electronically published on January 19, 2005

\title{
A GLOBAL STABILITY CRITERION FOR A FAMILY OF DELAYED POPULATION MODELS
}

\author{
BY
}

EDUARDO LIZ (Departamento de Matemática Aplicada II, E.T.S.I. Telecomunicación, Universidad de Vigo, Campus Marcosende, 36280 Vigo, Spain),

MANUEL PINTO (Departamento de Matemáticas, Facultad de Ciencias, Universidad de Chile, Casilla 653, Santiago, Chile),

VICTOR TKACHENKO (Institute of Mathematics, National Academy of Sciences of Ukraine, Tereshchenkivs'ka str. 3, Kiev, Ukraine),

AND

SERGEI TROFIMCHUK (Instituto de Matemática y Física, Universidad de Talca, Casilla 747, Talca, Chile)

Abstract. For a family of single-species delayed population models, a new global stability condition is found. This condition is sharp and can be applied in both monotone and nonmonotone cases. Moreover, the consideration of variable or distributed delays is allowed. We illustrate our approach on the Mackey-Glass equations and the LasotaWazewska model.

1. Introduction. This work is motivated by the problem of finding a sharp global stability result for the Mackey-Glass equation with variable delay

$$
x^{\prime}(t)=-\delta x(t)+\frac{p x(t-\tau(t))}{1+\left(x(t-\tau(t))^{n}\right.} .
$$

Here $\tau: \mathbb{R} \rightarrow[0, h]$ and $n, h, p, \delta>0$. With constant delay $\tau(t) \equiv h$, this equation was proposed in 1977 by Mackey and Glass [15] as a model in hematopoiesis (blood cell production).

Eq. (1.1) is a particular case of the class of delay differential equations

$$
x^{\prime}(t)=-\delta x(t)+f^{*}(x(t-\tau(t))), x \in \mathbb{R}^{+} \stackrel{\text { def }}{=}[0,+\infty),
$$

Received January 15, 2004.

2000 Mathematics Subject Classification. Primary 34K20; Secondary 92D25.

Key words and phrases. Global stability, delay differential equations, Schwarz derivative, Mackey-Glass equations, Lasota-Wazewska model.

E-mail address: eliz@dma.uvigo.es

E-mail address: pintoj@abello.dic.uchile.cl

E-mail address: vitk@imath.kiev.ua

E-mail address: trofimch@inst-mat.utalca.cl 
where $\delta>0, \tau: \mathbb{R} \rightarrow[0, h]$ is piecewise continuous, and $f^{*} \in C\left(\mathbb{R}^{+}, \mathbb{R}^{+}\right)$is either strictly decreasing or unimodal. Concretely, we will consider that one of the following conditions holds:

(H1) $\left(f^{*}\right)^{\prime}(x)<0$ for all $x>0$.

(H2) $\left(f^{*}\right)^{-1}(0)=0, \liminf _{x \rightarrow 0^{+}} \frac{f^{*}(x)}{x}>\delta$ and $f^{*}: \mathbb{R}^{+} \rightarrow \mathbb{R}^{+}$has only one critical point $c$ (maximum).

Besides Eq. (1.1), the family (1.2) includes the celebrated Nicholson blowflies equation, the Mackey-Glass equations, and the Lasota-Wazewska delay equation (see [1]-13] and 16] for more references).

Both (H1) and (H2) are sufficient to assure the existence of a unique positive equilibrium for (1.2); we denote such a solution by $x(t) \equiv x^{*}$. By definition, the global attractivity of (1.2) means that $\lim _{t \rightarrow+\infty} x(t)=x^{*}$ for every nontrivial solution $x(t)$ of the equation.

Recently, we proved in [13] a sharp global stability result which is easy to apply to Eq. (1.2) with monotone nonlinearity when $f^{*}$ is dominated in some sense by a rational function. We will assume that one of the following conditions is satisfied:

(D1) $\left(f^{*}\left(y+x^{*}\right)-\delta x^{*}-r(y)\right) y>0$ for all $y>\max \left\{-x^{*},-1 / b\right\}, y \neq 0$,

(D2) $\left(f^{*}\left(-y+x^{*}\right)-\delta x^{*}+r(y)\right) y<0$ for all $y \in\left(-1 / b, x^{*}\right), y \neq 0$,

where $r(x)=a x /(1+b x)$, for some constants $a<0, b \geq 0$ (if $b=0$, by abuse of notation, $-1 / b$ means $-\infty)$.

We have the following consequence from [13, Theorem 1.1]:

Proposition 1.1. Assume that $f^{*}$ satisfies (H1) and either (D1) or (D2). Then (1.2) is globally attracting if

$$
\delta e^{-h \delta}>-a \ln \frac{a^{2}-a \delta}{a^{2}+\delta^{2}} .
$$

As we show in Section 3 below, when (H1) holds, one of two conditions (D1), (D2) is always satisfied with $a=\left(f^{*}\right)^{\prime}\left(x^{*}\right)$ if $f^{*}$ has negative Schwarz derivative

$$
\left(S f^{*}\right)(x)=\frac{\left(f^{*}\right)^{\prime \prime \prime}(x)}{\left(f^{*}\right)^{\prime}(x)}-\frac{3}{2}\left(\frac{\left(f^{*}\right)^{\prime \prime}(x)}{\left(f^{*}\right)^{\prime}(x)}\right)^{2}
$$

for all $x>0$. Both the Lasota-Wazewska equation and the Mackey-Glass equation with decreasing nonlinearity satisfy all conditions of Proposition 1.1. Hence, (1.3) gives a stability condition which improves the main theorems in [3, 6, 11]. Now, the importance of Proposition 1.1 relies on its sharp nature: for every monotone $f^{*}$ satisfying (D1) or (D2) and for every triple $a<0, \delta>0, h>0$ which does not satisfy (1.3), the equilibrium $x(t)=x^{*}$ loses its asymptotical stability with an appropriate choice of piecewise-continuous periodic delay function $\tau: \mathbb{R} \rightarrow[0, h]$. (See [8, 13] for details.)

However, the results in [13] do not apply directly to the nonmonotone case of Eq. (1.2). The main obstacle for this consists in the fact that unimodal functions do not satisfy the negative feedback condition with respect to the level $x=x^{*}$. A simple approach allowing us (at least theoretically) to overcome this difficulty was proposed in [13]: it suffices to prove that $f$ has negative feedback on some interval covering the projection $\pi(\mathcal{A})$ of the global attractor $\mathcal{A}$ of (1.2) on $\mathbb{R}^{+}$(here $\pi: C([-h, 0] ; \mathbb{R}) \rightarrow \mathbb{R}$ is defined by $\left.\pi(\phi)=\phi(0)\right)$. 
To apply this method, we should be able to obtain sufficiently sharp a priori estimations of the global attractivity domain for Eq. (1.2), and this can be a very difficult task. In the case of Nicholson's blowflies equation,

$$
x^{\prime}(t)=-\delta x(t)+p x(t-\tau(t)) e^{-\gamma x(t-\tau(t))}, \delta, p, \gamma>0,
$$

we succeeded to solve it (see [13]), while in the case of Eq. (1.1) this task seems to be unrealizable (due to the necessity of enormous computations).

The main theorem in this paper allows us to deal easily with unimodal nonlinearities, even when the negative feedback condition does not hold. Hence, in Section 3 we are able to apply our result not only to the most famous examples of equation (1.2) with variable delay, but also for the case of distributed or multiple delays, improving the results in [3, 4, 6, 10, 11, 12, 16. For the reader's convenience, we state the main result in Section 2 , and we prove it in Section 4, just after the section containing the applications.

2. The main result. Let $C:=C([-h, 0] ; \mathbb{R})$ be the space of continuous functions from $[-h, 0]$ to $\mathbb{R}, h>0$, equipped with the sup norm $\|\varphi\|=\max _{-h \leq \theta \leq 0}|\varphi(\theta)|$. We denote by $C^{+}$the cone of nonnegative functions $C^{+}=\{\phi \in C: \phi \geq 0\}$.

We assume that the functional $f: \mathbb{R} \times C^{+} \rightarrow \mathbb{R}^{+}$satisfies the Carathéodory conditions and that, for every constant element $m(s) \equiv m$ from $C^{+}$, the function $f(\cdot, m): \mathbb{R} \rightarrow \mathbb{R}^{+}$ is constant almost everywhere on $\mathbb{R}$. Thus we obtain a continuous map $f^{*}: \mathbb{R}^{+} \rightarrow \mathbb{R}^{+}$ such that $f^{*}(m)=f(t, m)$ for all $m \in \mathbb{R}^{+}$and for a.e. $t \in \mathbb{R}$.

We also assume that $f$ satisfies the following condition:

$$
m=\inf _{[-h, 0]} \psi(t) \leq \sup _{[-h, 0]} \psi(t)=M \text { implies } f(t, \psi) \in f^{*}([m, M]), t \in \mathbb{R} .
$$

Notice that each one of hypotheses (H1), (H2) implies immediately the existence of a unique positive equilibrium $x(t) \equiv x^{*}$ of the delay differential equation

$$
x^{\prime}(t)=-\delta x(t)+f\left(t, x_{t}\right), x \in \mathbb{R}^{+} .
$$

Now we are ready to state the main result of this paper

Theorem 2.1. Assume that the conditions (1.3), 2.1), (Hk) and (D $j)$ are satisfied for some choice of $j, k \in\{1,2\}$. Then $\lim _{t \rightarrow+\infty} x(t)=x^{*}$ for every nonzero solution $x(t)$ of equation (2.2).

Remark 2.2. As was noticed in the paragraph below Proposition 1.1, Theorem 2.1 is sharp within the class of equations (2.2) with $f$ satisfying $(\mathrm{H} k)$ and $(\mathrm{D} j)$ for some choice of $j, k \in\{1,2\}$.

The following corollary will be useful in the applications.

Corollary 2.3. Assume that $f^{*}$ satisfies either (H1) or (H2), has negative Schwarz derivative for all $x>0, x \neq c$, and $a=\left(f^{*}\right)^{\prime}\left(x^{*}\right)<0$. If, in addition, conditions (1.3) and (2.1) hold, then $\lim _{t \rightarrow+\infty} x(t)=x^{*}$ for every nonzero solution $x(t)$ of equation (2.2).

Proof. By Lemma 2.1 in [14], condition (D1) with $b=\left(f^{*}\right)^{\prime \prime}\left(x^{*}\right)$ holds if $\left(f^{*}\right)^{\prime \prime}\left(x^{*}\right)>0$. If $\left(f^{*}\right)^{\prime \prime}\left(x^{*}\right)<0$, condition (D2) holds with $b=-\left(f^{*}\right)^{\prime \prime}\left(x^{*}\right)$. Finally, if $\left(f^{*}\right)^{\prime \prime}\left(x^{*}\right)=0$, 
then $x^{*}$ is an inflexion point and (D1) holds (notice that in this case $r(x)=-a x$ is a linear function).

3. Applications. In this section we apply Corollary 2.3 to obtain global stability results for some well-known delayed population models. First, we observe that the direct application of this corollary to Nicholson's blowflies equation (1.4) gives Theorem 2.1 in [13. Next we consider other examples.

3.1. Lasota-Wazewska model with distributed delay. Our first example is a generalization of the celebrated model proposed by Wazewska and Lasota in 1976 for the survival of red blood cells (see, for example, [6, 10, 11, 12, 16]),

$$
x^{\prime}(t)=-\delta x(t)+p e^{-\gamma x(t-h)}, t \geq 0,
$$

where $\delta, p, \gamma, h>0$. This generalization is of the form

$$
x^{\prime}(t)=-\delta x(t)+\int_{0}^{h} e^{-\gamma x(t-s)} d q(s), t \geq 0,
$$

where $\delta, \gamma>0$, and $q$ is a nondecreasing and nonnegative function defined on $[0, h]$, with $\int_{0}^{h} d q(s)=p>0$.

Function $f^{*}$ is defined by $f^{*}(x)=p e^{-\gamma x}$, which satisfies $(\mathrm{H} 1)$, and $\left(S f^{*}\right)(x)=$ $-\gamma^{2} / 2<0$ for all $x>0$.

The unique positive equilibrium $x^{*}$ of (3.2) is the unique positive root of equation $f^{*}(x)=\delta x$. Since $\left(f^{*}\right)^{\prime}\left(x^{*}\right)=-\gamma \delta x^{*}<0$, an application of Corollary 2.3 gives the following result:

TheOREM 3.1. The unique positive equilibrium $x^{*}$ of (3.2) is a global attractor if the following condition holds:

$$
e^{-\delta h}>\gamma x^{*} \ln \left(\frac{\left(\gamma x^{*}\right)^{2}+\gamma x^{*}}{1+\left(\gamma x^{*}\right)^{2}}\right) .
$$

In the particular case of equation (3.1), Li and Chang proved recently 11, Theorem $2]$ the global attractivity of $x^{*}$ under condition $\gamma x^{*}\left(1-e^{-\delta h}\right) \leq 1+e^{-\delta h} /\left(\gamma x^{*}\right)$, or, equivalently,

$$
e^{-\delta h} \geq \gamma x^{*} \frac{\gamma x^{*}-1}{1+\left(\gamma x^{*}\right)^{2}} .
$$

It is easy to check that Theorem 3.1 improves this result. Indeed, for $\gamma x^{*} \leq 1$ both conditions hold. Now, for $\gamma x^{*}>1$, 3.3) is sharper than (3.4) since for $y>0$ we have the inequality $\ln (1+y)<y$ (take $\left.y=\left(\gamma x^{*}-1\right) /\left(1+\left(\gamma x^{*}\right)^{2}\right)\right)$. We notice that condition (3.4) was already obtained in 6]. Finally, for Eq. (3.2), Theorem 3.1 improves the result in [12, Corollary 4.8].

3.2. Mackey-Glass model with unimodal nonlinearity. Our next example is the MackeyGlass equation (1.1). Taking into account the form of the right-hand side of (1.1), it has only one constant solution $x(t)=0$ if $p \leq \delta$ and two constant solutions $x(t)=0$ and $x(t)=x^{*}=(p / \delta-1)^{1 / n}$ in the case $p>\delta$.

TheOrEm 3.2. Let $p>\delta$. Then the positive equilibrium of Eq. (1.1) is a global attractor if one of the following three conditions holds: 
(i) $n \in(0,2]$;

(ii) $n>2$ and $\delta \in\left(0, \delta_{1}(n, h)\right]$, where

$$
\delta_{1}(n, h)=\frac{1}{h} \ln \left(\frac{n^{2}-2 n+2}{n^{2}-3 n+2}\right) ;
$$

(iii) $n>2, \delta>\delta_{1}(n, h)$, and

$$
-\frac{\delta}{1-n+\delta n / p} \exp (-h \delta)>\ln \frac{(1-n+\delta n / p)^{2}-(1-n+\delta n / p) \delta}{\delta^{2}+(1-n+\delta n / p)^{2}} .
$$

Proof. See [3] for the cases (i), (ii) and when $n>2, p / \delta \leq n /(n-1)$. Hence we have to work only with $n>2, p / \delta>n /(n-1)$. In this case, it is easy to check that the nonlinearity $f^{*}(x)=p x /\left(1+x^{n}\right)$ in (1.1) satisfies condition (H2), with a unique critical point $c=(n-1)^{-1 / n}$. Moreover, $f^{*}$ has negative Schwarz derivative for all $x \neq c$ (e.g. see [3]), and $\left(f^{*}\right)^{\prime}\left(x^{*}\right)<0$. An application of Corollary 2.3 ends the proof.

REmark 3.3. We emphasize that Theorem 3.2 improves the results in [3, where some comparisons with previous results in the literature can be found. Moreover, condition (3.5) is sharp in the class of equations (1.1) with $\tau(t) \leq h$.

3.3. Mackey-Glass model with distributed delay. Consider the following generalization of the Mackey-Glass equation [4]:

$$
x^{\prime}(t)=-\delta x(t)+\int_{0}^{h} \frac{d q(s)}{1+x^{n}(t-s)}, t>0, x \geq 0,
$$

with $\delta, n>0$. The nonconstant, nondecreasing and nonnegative function $q(s)$ is defined on $[0, h]$ and $\int_{0}^{h} d q(s)=p>0$.

Function $f^{*}$ is defined by $f^{*}(x)=p /\left(1+x^{n}\right)$, which satisfies $(\mathrm{H} 1)$ and $\left(S f^{*}\right)(x)=$ $\left(1-n^{2}\right) /\left(2 x^{2}\right)<0$ for all $x>0$, if $n>1$.

The unique positive equilibrium $x^{*}$ of (3.6) is the unique positive root of equation

$$
x^{n+1}+x=\frac{p}{\delta} .
$$

Theorem 3.4. The unique positive equilibrium $x^{*}$ of (3.6) is a global attractor if one of the following conditions holds:

(i) $n \in(0,1]$;

(ii) $n>1$ and $\delta \in\left(0, \delta_{2}(n, h)\right]$, where

$$
\delta_{2}(n, h)=\frac{1}{h} \ln \left(\frac{n^{2}+1}{n^{2}-n}\right) ;
$$

(iii) $n>1, \delta>\delta_{2}(n, h)$, and

$$
\exp (-h \delta)>c \ln \frac{c^{2}+c}{1+c^{2}}, \quad c=\frac{\delta n\left(x^{*}\right)^{n+1}}{p} .
$$

Proof. The cases (i) and (ii) were proved in 3]. Since $\left(f^{*}\right)^{\prime}\left(x^{*}\right)=-\delta^{2} n\left(x^{*}\right)^{n+1} / p$, case (iii) follows from Corollary 2.3 
REMARK 3.5. We notice that condition (3.7) holds for all values of $h>0$ if $c \leq 1$. This is equivalent (see [12 Theorem 4.10]) to condition

$$
p(n-1)^{(n+1) / n} \leq \delta n .
$$

It was proved in 12 that $(3.8)$ is the best possible delay independent condition for (3.6).

4. Proof of the main result. In this section we prove Theorem 2.1 For this, we first show that, after some transformations, Equation (2.2) and the set of hypotheses (D1)-(D2) can be simplified for the case $b>0$. Then, we adapt the arguments in [13] to complete the proof. We will asssume that $b>0$ until the end of Section 4 , where the case $b=0$ is considered.

4.1. A reduction. First, we transform (2.2), making the changes of variables $s=\delta t$ and $x=b^{-1} y$. These changes do not affect the global stability property of the equilibrium solution of Eq. (2.2), which now has the following simpler form:

$$
x^{\prime}(t)=-x(t)+\widetilde{f}\left(t, x_{t}\right), x \in \mathbb{R}^{+},
$$

where $\tilde{f}$ satisfies either (D1) or (D2) with the same $a<0$ and $b=1$. In the sequel, by abuse of notation, we shall use the same letter $f$ for $\tilde{f}$. Using the variation of constants formula, we can check that every solution of (4.1) is nonnegative, if it takes nonnegative values on the initial interval. Hence, if we consider a nontrivial solution $x(t)$ and denote $M=\lim \sup _{t \rightarrow \infty} x(t), m=\liminf _{t \rightarrow \infty} x(t)$, we conclude that $m \geq 0$. However, we will need stronger positivity properties of the solutions to (4.1):

TheOREm 4.1. If either (H1) or (H2) holds, then all solutions $x(t)$ of (4.1) are defined in $[0,+\infty)$. Moreover, there exist $\nu, \mu>0$ such that $\mu \leq m \leq M \leq \nu$ for each eventually nontrivial $x(t)$. Furthermore, $[m, M] \subseteq f^{*}([m, M])$.

Proof. See Lemmas 3.1, 3.3 and Theorem 3.6 in 12 (their proofs are still valid for the nonautonomous case under condition (2.10).

The following result shows that, from now on, we should focus our analysis solely on solutions which oscillate around the positive equilibrium:

Lemma 4.2. If $m \leq M \leq x^{*}$ or $M \geq m \geq x^{*}$, then $\lim _{t \rightarrow \infty} x(t)=x^{*}$.

Proof. We have from Theorem 4.1 that $[m, M] \subseteq f^{*}([m, M])$, where $M \geq m \geq x^{*}$ or $m \leq M \leq x^{*}$. Since $f^{*}$ is either decreasing or unimodal, this is possible only if $m=M=x^{*}$.

Now let $x(t)$ be such that $M>x^{*}>m$. Then there exist two sequences of intervals $\left(a_{j}, b_{j}\right)$ and $\left(a_{j}^{\prime}, b_{j}^{\prime}\right)$ with the following properties: $x\left(a_{j}\right)=x\left(b_{j}\right)=x\left(a_{j}^{\prime}\right)=x\left(b_{j}^{\prime}\right)=x^{*}$, $x(t)>x^{*}$ for $t \in\left(a_{j}, b_{j}\right)$ and $x(t)<x^{*}$ for $t \in\left(a_{j}^{\prime}, b_{j}^{\prime}\right)$. Furthermore, $\max _{\left(a_{j}, b_{j}\right)} x(t) \rightarrow M$ and $\min _{\left(a_{j}^{\prime}, b_{j}^{\prime}\right)} x(t) \rightarrow m$ as $j \rightarrow \infty$.

Lemma 4.3. If $t_{j}$ is the leftmost point from $\left(a_{j}, b_{j}\right)$ satisfying $x\left(t_{j}\right)=\max _{\left(a_{j}, b_{j}\right)} x(t)$, then $t_{j}-a_{j} \leq h$. Analogously, if $s_{j}$ is the leftmost point from $\left(a_{j}^{\prime}, b_{j}^{\prime}\right)$ satisfying $x\left(s_{j}\right)=$ $\min _{\left(a_{j}^{\prime}, b_{j}^{\prime}\right)} x(t)$, then $s_{j}-a_{j}^{\prime} \leq h$. 
Proof. In the proof, $c^{*}$ denotes $\min \left\{\left(f^{*}\right)^{-1}\left(x^{*}\right)\right\}$ in the unimodal case, and $c^{*}=0$ if $f^{*}$ is decreasing. We have $x^{\prime}\left(t_{j}\right)=0$ and $f\left(t_{j}, x_{t_{j}}\right)=x\left(t_{j}\right)>x^{*}$. If $x\left(t_{j}+s\right)>x^{*}$ holds for all $s \in[-h, 0]$, then $x^{*}<f\left(t_{j}, x_{t_{j}}\right) \in f^{*}\left(\left[x^{*}, M\right]\right)$, a contradiction (since $f$ is decreasing on $\left.\left[x^{*},+\infty\right)\right)$.

Analogously, $x^{\prime}\left(s_{j}\right)=0$ so that $f\left(s_{j}, x_{s_{j}}\right)=x\left(s_{j}\right)<x^{*}$. If we suppose that $c^{*}<$ $x\left(s_{j}+s\right)<x^{*}$ for $s \in[-h, 0]$, then we get a contradiction again, since the relations $x^{*}>f\left(t_{j}, x_{t_{j}}\right) \in f^{*}\left(\left[c^{*}, x^{*}\right]\right) \subset\left[x^{*},+\infty\right)$ are impossible. Thus either $x\left(s_{j}+s^{\prime}\right) \geq x^{*}$ for some $s^{\prime} \in[-h, 0]$ (and the lemma is proved) or $x\left(s_{j}+s\right)<x^{*}$ for all $s \in[-h, 0]$ and $x\left(s_{j}+s^{\prime \prime}\right) \leq c^{*}$ for some $s^{\prime \prime} \in[-h, 0]$. The second case is only possible if $f^{*}$ is unimodal, and we can suppose that $x\left(s_{j}+s^{\prime \prime}\right)=\min _{s \in[-h, 0]} x\left(s_{j}+s\right) \leq c^{*}$. Thus

$$
x\left(s_{j}\right)=f\left(s_{j}, x_{s_{j}}\right) \geq f^{*}\left(x\left(s_{j}+s^{\prime \prime}\right)\right)>x\left(s_{j}+s^{\prime \prime}\right),
$$

contradicting the choice of $x\left(s_{j}\right)$ as the minimal value of $x(t)$ on $\left(a_{j}^{\prime}, b_{j}^{\prime}\right)$.

A simple observation of the graph of $f=f^{*}$ shows that, in the case (H1), the interval $I=[0, f(0)]$ is invariant under $f$; moreover, $f^{2}(I) \subset \operatorname{Int}(I)$. In the case (H2), if we take a sufficiently small $\epsilon>0$ and consider $I=[-\epsilon+f(f(c)+\epsilon), f(c)+\epsilon]$, we again obtain that $f(I) \subset \operatorname{Int}(I)$. Furthermore, Theorem 4.1 implies that $[m, M] \subset \operatorname{Int}(I)$. When $f$ is decreasing, this relation follows from the chain of inclusions $[m, M] \subset f([m, M]) \subset$ $f^{3}\left(\mathbb{R}^{+}\right)=f^{2}(I) \subset \operatorname{Int}(I)$. In the unimodal case, if we take a sufficiently large integer $N$, we get analogously $[m, M] \subset f([m, M]) \subset f^{N}([m, M]) \subset f(I) \subset \operatorname{Int}(I)$. As a consequence, if we analyze the asymptotical behavior of some fixed solution $x(t)$ to (4.1), we do not need to know the values $f(t, \phi)$ for those $\phi$ whose graphs do not lie entirely within the rectangle $[-h, 0] \times I$. In particular, we can replace the original nonlinearity $f(t, \phi)$ with $f_{1}(t, \phi)=f(t, T \phi)$, where the squeezing operator $T: C^{+} \rightarrow C^{+}$acts as follows:

$$
(T \phi)(s)= \begin{cases}\phi(s) & \text { if } \phi(s) \in I, \\ \max I & \text { if } \phi(s)>\max I, \\ \min I & \text { if } \phi(s)<\min I .\end{cases}
$$

Obviously, $f_{1}(t, \phi)$ satisfies (2.1) and $f_{1}^{*}(x)=f^{*}(x)$ for all $x \in I$. Also, $f_{1}^{*}$ is strictly positive and continuous on the whole $\mathbb{R}$ and it is a constant function outside of $I$. An elementary analysis shows that $f_{1}^{*}$ and $f^{*}$ satisfy the same hypothesis from the list of (D1) and (D2).

Summing up all the above, we come to the following conclusion: in order to establish the global stability of Eq. (4.1), it suffices to prove the convergence to $x^{*}$ of every solution $x(t)$ of

$$
x^{\prime}(t)=-x(t)+f_{1}\left(t, x_{t}\right), x \in \mathbb{R} .
$$

Due to Lemmas 4.2 and 4.3, we can suppose additionally that $x(t)$ oscillates about $x^{*}$ and satisfies the conclusions of Lemma 4.3 Notice that $x(t) \equiv x^{*}$ is the unique steady state solution of (4.2).

We can simplify the situation even more if we translate the origin to the equilibrium point $x^{*}$. It can be done by the simple shift $x=y+x^{*}$ in the case (D1) and by the transformation $x=x^{*}-y$ if $f_{1}^{*}$ satisfies (D2). As a result, (4.2) is transformed into the 
equation

$$
x^{\prime}(t)=-x(t)+g\left(t, x_{t}\right), x \in \mathbb{R},
$$

whose unique equilibrium is the trivial solution $x(t) \equiv 0$. Now, we are interested in proving the convergence to zero of every solution $x(t)$ of (4.3) which oscillates about 0 and satisfies the conclusions of Lemma 4.3. Since $g(t, \phi)=f_{1}\left(t, \phi+x^{*}\right)-x^{*}$ in the case (D1) and $g(t, \phi)=-f_{1}\left(t,-\phi+x^{*}\right)+x^{*}$ in the case (D2), it is easy to check that the function $g^{*}(y)=g(t, y), y \in \mathbb{R}$, is nonincreasing or unimodal (notice that for (D2) the critical point in case of unimodality is a local minimum instead of a maximum). Furthermore, $g^{*}$ satisfies (2.1) and the following modification of (D1):

(DD) $\left(g^{*}(y)-r(y)\right) y>0$ for all $y>-1, y \neq 0$,

where $r(y)=a y /(1+y)$. It should be noticed that, in general, the negative feedback condition on $g^{*}$ is not assumed.

Let $y(t)$ be a solution of (4.3) such that

$$
\liminf _{t \rightarrow \infty} y(t)=m<0<M=\limsup _{t \rightarrow \infty} y(t) .
$$

Then there exist two sequences of intervals $\left(a_{j}, b_{j}\right)$ and $\left(a_{j}^{\prime}, b_{j}^{\prime}\right)$ such that $y\left(a_{j}\right)=y\left(b_{j}\right)=$ $y\left(a_{j}^{\prime}\right)=y\left(b_{j}^{\prime}\right)=0, y(t)>0$ for $t \in\left(a_{j}, b_{j}\right)$ and $y(t)<0$ for $t \in\left(a_{j}^{\prime}, b_{j}^{\prime}\right)$. Moreover, for $y(t)$, the conclusions of Lemma 4.3 are fulfilled: if $t_{j}$ is the leftmost point from $\left(a_{j}, b_{j}\right)$ satisfying $y\left(t_{j}\right)=\max _{\left(a_{j}, b_{j}\right)} y(t)$, then $t_{j}-a_{j} \leq h$. Analogously, if $s_{j}$ is the leftmost point from $\left(a_{j}^{\prime}, b_{j}^{\prime}\right)$ satisfying $y\left(s_{j}\right)=\min _{\left(a_{j}^{\prime}, b_{j}^{\prime}\right)} y(t)$, then $s_{j}-a_{j}^{\prime} \leq h$. We can also assume that $M_{j}=y\left(t_{j}\right) \rightarrow M$ and $m_{j}=y\left(s_{j}\right) \rightarrow m$ as $j \rightarrow \infty$.

The following consequence of Lemma 4.3 will play a key role in the proof of Theorem 2.1 .

Corollary 4.4. Let $\widetilde{m}(u)=\min _{\theta \in[-h, 0]} y(u+\theta), \widetilde{M}(u)=\max _{\theta \in[-h, 0]} y(u+\theta)$. Then for sufficiently large $j$ :

$$
g\left(u, y_{u}\right)>r(\widetilde{M}(u)), \quad u \in\left(a_{j}^{\prime}, s_{j}\right]
$$

and, if $\widetilde{m}(u)>-1$,

$$
g\left(u, y_{u}\right)<r(\widetilde{m}(u)), \quad u \in\left(a_{j}, t_{j}\right]
$$

Proof. We prove (4.4); the proof of (4.5) is similar.

Since $g\left(u, y_{u}\right) \in g^{*}([\widetilde{m}(u), \widetilde{M}(u)])$, we get

$$
g\left(u, y_{u}\right) \geq \min _{s \in[\widetilde{m}(u), \widetilde{M}(u)]} g^{*}(s) .
$$

Hence the proof of (4.4) is immediate for $g^{*}$ decreasing on $[\widetilde{m}(u), \widetilde{M}(u)]$.

If $g^{*}$ is unimodal with a local minimum at some point $c^{\prime} \in[\widetilde{m}(u), \widetilde{M}(u)]$, then $g\left(u, y_{u}\right) \geq g^{*}\left(c^{\prime}\right)>r\left(c^{\prime}\right) \geq r(\widetilde{M}(u)$. Notice that, in view of our reduction, the point $c^{\prime}$ of local minimum is necessarily positive.

Next, for $g^{*}$ having a local maximum at some point $c$ (it must be negative) we will prove that, for sufficiently large $j$,

$$
g^{*}(\widetilde{m}(u)) \geq m_{j} \geq g^{*}\left(\widetilde{M}\left(s_{j}\right)\right) \geq g^{*}(\widetilde{M}(u)), u \in\left(a_{j}^{\prime}, s_{j}\right],
$$


From (4.6) we easily deduce (4.4):

$$
g\left(u, y_{u}\right) \in\left[\min \left\{g^{*}(\widetilde{m}(u)), g^{*}(\widetilde{M}(u))\right\}, g^{*}(c)\right] \subset\left[r(\widetilde{M}(u)), g^{*}(c)\right] .
$$

Since, by definition, $y(t)<0$ for $t \in\left(a_{j}^{\prime}, s_{j}\right]$, the following inequalities are fulfilled for $u \in\left[a_{j}^{\prime}, s_{j}\right]:$

$$
\widetilde{M}(u) \geq \widetilde{M}\left(s_{j}\right) \geq 0, g^{*}\left(\widetilde{M}\left(s_{j}\right)\right) \geq g^{*}(\widetilde{M}(u)) .
$$

To prove the first inequality in (4.6), we observe that, for $u \in\left[a_{j}^{\prime}, s_{j}\right]$, either $\widetilde{m}(u)=$ $y(u) \geq m_{j}$, or $\widetilde{m}(u)=y(u-h)<m_{j}$, or $\widetilde{m}(u)=y\left(s_{k}\right)$ for some $k \leq j$. In the first case, we have $g^{*}(\widetilde{m}(u)) \geq \widetilde{m}(u) \geq m_{j}$ which proves the leftmost inequality of (4.6). In the other cases, $c \geq \widetilde{m}(u) \geq y\left(s_{k}\right)=m_{k}$ for some $k \leq j$. Since $m=\lim _{j \rightarrow \infty} m_{j}=\liminf _{t \rightarrow \infty} y(t)$, there exist $\varepsilon>0, N_{0} \in \mathbb{N}$ such that

$$
m_{r}<\min g^{*}\left(\left[m_{k}-\varepsilon, m_{k}\right]\right) \text {, for all } r, k \geq N_{0} .
$$

In this way, $g^{*}(\widetilde{m}(u)) \geq g^{*}\left(m_{k}\right)>m_{j}$ when $j$ is sufficiently large.

It remains to prove the inequality

$$
m_{j} \geq g^{*}\left(\widetilde{M}\left(s_{j}\right)\right) .
$$

Since

$$
m_{j}=g\left(s_{j}, y_{s_{j}}\right) \in g^{*}\left(\left[\widetilde{m}\left(s_{j}\right), \widetilde{M}\left(s_{j}\right)\right]\right)=\left[\min \left\{g^{*}\left(\widetilde{m}\left(s_{j}\right)\right), g^{*}\left(\widetilde{M}\left(s_{j}\right)\right)\right\}, g^{*}(c)\right],
$$

inequality (4.8) holds if $g^{*}\left(\widetilde{M}\left(s_{j}\right)\right) \leq g^{*}\left(\widetilde{m}\left(s_{j}\right)\right)$. Assume the contrary; then, $m_{j}=$ $g\left(s_{j}, y_{s_{j}}\right) \in\left[g^{*}\left(\widetilde{m}\left(s_{j}\right)\right), g^{*}(c)\right] \subset\left[g^{*}\left(m_{k}\right), g^{*}(c)\right]$, for some $k \leq j$. Hence $m_{j} \geq g^{*}\left(m_{k}\right)$, a contradiction to (4.7).

4.2. Domains of parameters. Based on the previous subsection, we will use the rational function $r(x)=a x /(1+x)$ and we will prove Theorem 2.1 for equation

$$
x^{\prime}(t)=-x(t)+f\left(t, x_{t}\right),
$$

where $f^{*}(x)=f(t, x), x \in \mathbb{R}$, satisfies the inequality

$$
\left(f^{*}(x)-r(x)\right) x>0 \text { for } x>-1, x \neq 0 .
$$

Inequality (1.3) takes the form

$$
\theta=e^{-h}>-a \ln \frac{a^{2}-a}{a^{2}+1} .
$$

Following [13], in order to prove Theorem 1.1, we decompose the domain of parameters $(a, \theta) \in(-\infty, 0) \times(0,1)$ satisfying (4.11) into two complementary subsets:

$$
\begin{gathered}
\mathcal{B}=\left\{(a, \theta): \frac{a^{2}+a}{a^{2}+1}<\theta<1\right\} ; \\
\mathcal{D}=\left\{(a, \theta):-a \ln \frac{a^{2}-a}{a^{2}+1}<\theta \leq \frac{a^{2}+a}{a^{2}+1}\right\} .
\end{gathered}
$$

Notice that $\mathcal{B}$ contains all points $(a, \theta) \in[-1,0) \times(0,1)$. We will also split the domain $\mathcal{D}$ into two subsets $\mathcal{D}^{*}$ and $\mathcal{S}$ defined by

$$
\mathcal{S}=\left\{(a, \theta) \in \mathcal{D}: \quad \theta \in[0.8,1), \frac{95 a+108}{5(19 a-5)} \leq \theta \leq \frac{a^{2}+a}{a^{2}+1}\right\}, \mathcal{D}^{*}=\mathcal{D} \backslash \mathcal{S} .
$$


4.3. Global stability for $(a, \theta) \in \mathcal{B}$. We again use the notation

$$
M=\lim \sup _{t \rightarrow \infty} x(t), m=\lim \inf _{t \rightarrow \infty} x(t),
$$

and, taking into account Lemma 4.2 we only consider the case $m<0<M$. As before, let $\left\{t_{j}\right\}$ and $\left\{s_{j}\right\}$ be two sequences of points of local maximum and local minimum such that $x\left(t_{j}\right)=M_{j} \rightarrow M$ and $x\left(s_{j}\right)=m_{j} \rightarrow m$ as $j \rightarrow \infty$.

First, we prove the global attractivity of Eq. (4.9) if $a(1-\theta)>-1$.

LEMmA 4.5. If $a(1-\theta)>-1$, then $M=m=\lim _{t \rightarrow \infty} x(t)=0$.

Proof. For $\varepsilon>0$ arbitrary, we have that $x(s) \leq M+\varepsilon$ and $x(s) \geq m-\varepsilon$ for $s$ sufficiently large. Next, by Lemma 4.3 there exists $h_{j} \in[0, h]$ such that $x\left(s_{j}-h_{j}\right)=0$. In view of Corollary 4.4, for $s \in\left(s_{j}-h_{j}, s_{j}\right]$,

$$
f\left(s, x_{s}\right)>r\left(\max _{\theta \in[-h, 0]} x(s+\theta)\right) \geq r(M+\varepsilon) .
$$

Therefore, by the variation of constants formula, we have

$$
m_{j}=x\left(s_{j}\right)=\int_{s_{j}-h_{j}}^{s_{j}} e^{-\left(s_{j}-s\right)} f\left(s, x_{s}\right) d s>\left(1-e^{-h}\right) r(M+\varepsilon) .
$$

As a limit form of this inequality, we get

$$
m \geq(1-\theta) r(M) .
$$

Hence, $m>-1$. Analogously, taking into account (4.5),

$$
\begin{aligned}
M_{j} & =x\left(t_{j}\right)=\int_{t_{j}-h_{j}^{\prime}}^{t_{j}} e^{-\left(t_{j}-s\right)} f\left(s, x_{s}\right) d s \\
& <\left(1-e^{-h}\right) r\left(\min _{\theta \in[-h, 0]} x(s+\theta)\right) \leq(1-\theta) r(m-\varepsilon),
\end{aligned}
$$

where the number $h_{j}^{\prime} \in[0, h]$ is such that $x\left(t_{j}-h_{j}^{\prime}\right)=0$. Therefore,

$$
M \leq(1-\theta) r(m)
$$

By (4.12) and (4.13), we have

$$
M \leq(1-\theta) r((1-\theta) r(M)),
$$

which is equivalent to

$$
\frac{M(1+a(1-\theta))(1-a(1-\theta)+M)}{1+M(1+a(1-\theta))} \leq 0 .
$$

Since $a(1-\theta)>-1$, the last inequality is possible only if $M=0$.

Now, in Lemma 4.7 given below, we prove the global attractivity of Eq. (4.9) for all parameters $(a, \theta) \in \mathcal{B}$. In the proof of Lemma 4.7 we use the following statement:

Lemma 4.6 ([13, Proposition 3.3]). Let $q:[\alpha, \beta] \rightarrow[\alpha, \beta]$ be a $C^{3}$ map with a unique fixed point $\gamma$ and with at most one critical point $x^{*}$ (maximum). If $\gamma$ is locally asymptotically stable and the Schwarzian derivative $(S q)(x)<0$ for all $x \neq x^{*}$, then $\gamma$ is the global attractor of $q$.

LEMmA 4.7. If $(a, \theta) \in \mathcal{B}$, then $M=m=\lim _{t \rightarrow \infty} x(t)=0$. 
Proof. Due to Lemma 4.5 we can suppose that $a(1-\theta) \leq-1$. There exist sequences of positive $\varepsilon_{j}^{*} \rightarrow 0$ and $\varepsilon_{j} \rightarrow 0$ such that $x(s)<M+\varepsilon_{j}^{*}$ for all $s \in\left[s_{j}-h, s_{j}\right]$ and $m_{j}<m+\varepsilon_{j}$. By Lemma 4.2 there exists $h_{j} \in[0, h]$ such that $x\left(s_{j}-h_{j}\right)=\max _{\theta \in[-h, 0]} x\left(s_{j}+\theta\right) \geq 0$. From Corollary 4.4 we get

$$
m_{j}=x\left(s_{j}\right)=f\left(s_{j}, x_{s_{j}}\right) \geq r\left(x\left(s_{j}-h_{j}\right)\right) .
$$

Next, by (4.12), $m \geq(1-\theta) r(M)>a$. Hence $m_{j}>a$ for $j$ sufficiently large and, therefore, $r^{-1}\left(m_{j}\right)$ is well defined, where $r^{-1}(x)=x /(a-x)$ is the inverse function of $r(x)$. From (4.14) we obtain the inequality $x\left(s_{j}-h_{j}\right) \geq r^{-1}\left(m_{j}\right)$. Next, by the variation of constants formula and (4.4), we have

$$
\begin{aligned}
m_{j} & =x\left(s_{j}\right)=e^{-h_{j}} x\left(s_{j}-h_{j}\right)+\int_{s_{j}-h_{j}}^{s_{j}} e^{-\left(s_{j}-s\right)} f\left(s, x_{s}\right) d s \\
& \geq e^{-h_{j}} r^{-1}\left(m_{j}\right)+r\left(M+\varepsilon_{j}^{*}\right)\left(1-e^{-h_{j}}\right) \\
& \geq e^{-h^{-1}}\left(m_{j}\right)+r\left(M+\varepsilon_{j}^{*}\right)\left(1-e^{-h}\right) .
\end{aligned}
$$

We denote $\psi(x)=x-\theta r^{-1}(x)$; as a limit form of 4.15), we get

$$
\psi(m) \geq(1-\theta) r(M) \geq a(1-\theta) .
$$

This implies that

$$
m \geq \frac{a(1-\theta)(a-m)}{a-m-\theta} .
$$

Next, since $(a, \theta) \in \mathcal{B}$, we have that $a^{2}(1-\theta)+a-\theta<0 \leq m(a(1-\theta)+1)$, from which

$$
\frac{a(1-\theta)(a-m)}{a-m-\theta}>-1 \text {. }
$$

From (4.17) and (4.18) it follows that $m>-1$, and hence we can use inequality (4.10) for $x(t)$ with sufficiently large $t$.

Analogously, by Lemma 4.2, there exists $h_{j}^{\prime} \in[0, h]$ such that

$$
x\left(t_{j}-h_{j}^{\prime}\right)=\min _{\theta \in[-h, 0]} x\left(s_{j}+\theta\right) \leq 0 .
$$

From (4.5) and (4.10) we get $x\left(t_{j}-h_{j}^{\prime}\right) \leq r^{-1}\left(M_{j}\right)$. By the variation of constants formula and (4.5), we have, for some positive sequence $\varepsilon_{j}^{*} \rightarrow 0$,

$$
\begin{aligned}
M_{j} & =x\left(t_{j}\right)=e^{-h_{j}^{\prime}} x\left(t_{j}-h_{j}^{\prime}\right)+\int_{t_{j}-h_{j}^{\prime}}^{t_{j}} e^{-\left(t_{j}-s\right)} f\left(s, x_{s}\right) d s \\
& \leq e^{-h_{j}^{\prime}} r^{-1}\left(M_{j}\right)+r\left(m-\varepsilon_{j}^{*}\right)\left(1-e^{-h_{j}^{\prime}}\right) \\
& \leq e^{-h} r^{-1}\left(M_{j}\right)+r\left(m-\varepsilon_{j}^{*}\right)\left(1-e^{-h}\right) .
\end{aligned}
$$

When $j \rightarrow \infty$, we obtain

$$
\psi(M) \leq(1-\theta) r(m) .
$$

Define $\chi(x)=\psi^{-1}((1-\theta) r(x))$. From (4.16) and (4.19) we conclude that $M \leq \chi(m)$ and $m \geq \chi(M)$. Hence, $[m, M] \subset \chi([m, M])$. On the other hand (see [13, Lemma 3.6]), $\chi:[\alpha, \beta] \rightarrow[\alpha, \beta]$ is strictly decreasing and $(S \chi)(x)<0$ for all $x \in[\alpha, \beta]$, where 
$\alpha=\chi(+\infty)=\psi^{-1}((1-\theta) a)>-1$ and $\beta=\chi(\alpha)$. Since, by our assumptions, $\chi^{\prime}(0)=$ $(1-\theta) a^{2} /(a-\theta) \in(-1,0)$, an application of Lemma 4.6] shows that 0 attracts $[\alpha, \beta]$ under $\chi$. Since $[m, M] \subseteq \chi^{k}([m, M]) \subseteq \chi^{k}([\alpha, \beta])$ for all integer $k \geq 1$, it is clear that $m=M=0$.

4.4. Global stability for $(a, \theta) \in \mathcal{D}$. In this subsection, we prove Theorem 2.1 for $(a, \theta) \in \mathcal{D}$. For this, we will use two scalar auxiliary functions introduced in [13].

For $(a, \theta) \in \mathcal{D}$, the inequality $a(\theta-1) / \theta-1>0$ holds, and therefore the interval $I=(-1, a(\theta-1) / \theta-1)$ is nonempty. Furthermore, $t_{1}=t_{1}(z)=-\ln \left(1-\frac{z}{r(z)}\right) \in[-h, 0]$ for every $z \in I \backslash\{0\}$. Consider now the map $F: I \rightarrow \mathbb{R}$ defined in the following way:

$$
F(z)= \begin{cases}0 & \text { if } z=0, \\ \min _{t \in[0, h]} y(t, z) & \text { if } z \in I \text { and } z>0, \\ \max _{t \in[0, h]} y(t, z) & \text { if } z \in(-1,0),\end{cases}
$$

where $y(t, z)$ is the solution of the initial value problem $y(s, z)=z, s \in\left[t_{1}(z)-\right.$ $\left.h, t_{1}(z)\right], z \in I$ for

$$
y^{\prime}(t)=-y(t)+r(y(t-h)) .
$$

The importance of function $F$ is due to the following result:

Lemma 4.8. Let $x(t)$ be a solution of (4.9) and set

$$
M=\limsup _{t \rightarrow \infty} x(t), \quad m=\liminf _{t \rightarrow \infty} x(t) .
$$

If $m, M \in I$, then $m \geq F(M)$ and $M \leq F(m)$.

Proof. Consider two sequences of extremal values $m_{j}=x\left(s_{j}\right) \rightarrow m, M_{j}=x\left(t_{j}\right) \rightarrow M$ such that $s_{j} \rightarrow+\infty, t_{j} \rightarrow+\infty$ as $j \rightarrow \infty$. Let $\varepsilon>0$ be such that $(m-\varepsilon, M+\varepsilon) \subset I$. Then $m_{j} \geq m-\varepsilon$ and $M_{j} \leq M+\varepsilon$ for sufficiently large $j$. We will prove that $m \geq F(M)$; the case $M \leq F(m)$ can be proved analogously by using inequality (4.5).

By Lemma 4.2 we can find $\tau_{j} \in\left[s_{j}-h, s_{j}\right)$ such that $x\left(\tau_{j}\right)=0$ and $x(s)<0$ for $s \in\left(\tau_{j}, s_{j}\right]$. Next, since $M+\varepsilon \in I, v_{j}=\tau_{j}+t_{1}(M+\varepsilon) \geq \tau_{j}-h$. We consider the solution $y(t)$ of Eq. (4.20) with initial condition $y(s)=M+\varepsilon, s \in\left[v_{j}-h, v_{j}\right]$, which satisfies $y\left(\tau_{j}\right)=0$. Obviously, $M+\varepsilon=y(t) \geq x(t)$ for all $t \in\left[v_{j}-h, v_{j}\right]$. Furthermore, for all $s \in\left[v_{j}, \tau_{j}\right]$, we have $\max _{\theta \in[-h, 0]} x(s+\theta) \leq M+\varepsilon$ so that, in view of (2.1) and (4.10), $f\left(s, x_{s}\right) \geq r(M+\varepsilon)$, and

$$
x(t)=\int_{\tau_{j}}^{t} e^{-(t-s)} f\left(s, x_{s}\right) d s \leq \int_{\tau_{j}}^{t} e^{-(t-s)} r(M+\varepsilon) d s=y(t), t \in\left[v_{j}, \tau_{j}\right],
$$

proving that $y(t) \geq x(t)$ for all $t \in\left[\tau_{j}-h, \tau_{j}\right]$.

Now, for $t \in\left[\tau_{j}, s_{j}\right]$, we have

$$
x(t)=\int_{\tau_{j}}^{t} e^{-(t-s)} f\left(s, x_{s}\right) d s \geq \int_{\tau_{j}}^{t} e^{-(t-s)} r(y(s-h)) d s=y(t),
$$

since, by (4.4) and (4.10),

$$
f\left(s, x_{s}\right) \geq r\left(\max _{\theta \in[-h, 0]} x(s+\theta)\right) \geq r\left(\max _{\theta \in[-h, 0]} y(s+\theta)\right)=r(y(s-h)) .
$$


Hence, by (4.21) and the definition of $F$, we have

$$
m_{j}=x\left(s_{j}\right) \geq y\left(s_{j}\right) \geq F(M+\varepsilon) .
$$

As a limit form of (4.22), we get $m \geq F(M)$.

When $(a, \theta) \in \mathcal{D}^{*}$, we need other auxiliary function; for $z \geq 0$, we define $F_{1}(z)$ $=\min _{t \in[0, h]} y(t, z)$, where $y(t, z)$ satisfies (4.20) and has the initial value $y(s, z)$ $=\left(1-e^{-s}\right) r(z), s \in[-h, 0]$.

Lemma 4.9. Suppose that $(a, \theta) \in \mathcal{D}$. Then $m \geq F_{1}(M)$.

Proof. Consider a solution $x(t)$ of (4.9) and take $\varepsilon, s_{j}, t_{j}, m_{j}, M_{j}, \tau_{j}$ as in the first two paragraphs of Lemma 4.8 ,

For $t \in\left[\tau_{j}-h, \tau_{j}\right]$, we have, by (2.1) and (4.10),

$$
x(t)=\int_{\tau_{j}}^{t} e^{-(t-u)} f\left(u, x_{u}\right) d u \leq \int_{\tau_{j}}^{t} e^{-(t-u)} r(M+\varepsilon) d u=y\left(t-\tau_{j}, M+\varepsilon\right) .
$$

Now, using the last inequality, (4.4) and (4.10), we get

$$
f\left(u, x_{u}\right) \geq r\left(\max _{\theta \in[-h, 0]} x(u+\theta)\right) \geq r\left(y\left(u-h-\tau_{j}, M+\varepsilon\right)\right), u \in\left[\tau_{j}, s_{j}\right] .
$$

This implies that

$$
\begin{aligned}
x\left(s_{j}\right) & =\int_{\tau_{j}}^{s_{j}} e^{-\left(s_{j}-u\right)} f\left(u, x_{u}\right) d u \geq \int_{\tau_{j}}^{s_{j}} e^{-\left(s_{j}-u\right)} r\left(y\left(u-h-\tau_{j}, M+\varepsilon\right)\right) d u \\
& =y\left(s_{j}-\tau_{j}, M+\varepsilon\right) \geq F_{1}(M+\varepsilon) .
\end{aligned}
$$

Since $\varepsilon>0$ and $x\left(s_{j}\right)=m_{j} \rightarrow m$ are arbitrary, we conclude that $m \geq F_{1}(M)$.

To finish the proof of Theorem 2.1, we need the following technical lemmas from [13]:

Lemma 4.10. Assume that $(a, \theta) \in \mathcal{D}$. Then $F(z)>\mathcal{R}(r(z)) \quad$ if $\quad z \in(0, a(\theta-1) / \theta-1)$, and $F(z)<\mathcal{R}(r(z))$ if $z \in\left((a \beta-1)^{-1}, 0\right)$, where

$$
\begin{aligned}
& \mathcal{R}(r)=\mathcal{R}(r, a, \theta)=\frac{\alpha(a, \theta) r}{1-\beta(a, \theta) r}, \\
& \alpha(a, \theta)=(1-a) \exp (\theta / a)+a>0, \\
& \beta(a, \theta)=-\frac{a^{2}+\exp (\theta / a)(1-2 a+2 \theta(a-1))-(1-a)^{2} \exp (\theta / a)}{a^{2}+\left(a-a^{2}\right) \exp (\theta / a)}>0 .
\end{aligned}
$$

Moreover, $\mathcal{R}(a)>-1$.

Lemma 4.11. If $(a, \theta) \in \mathcal{D}^{*}$ and $z \geq a(\theta-1) / \theta-1$, then $F_{1}(z)>\mathcal{R}(r(z))$.

Lemma 4.12. If $(a, \theta) \in \mathcal{S}$ and $z>0$, then

$$
F_{1}(z)>\frac{1+\ln \theta-\theta}{2+\ln \theta-\theta} \frac{a r(z)}{1+r(z) \frac{1+\ln \theta-\theta}{1-\theta}} \stackrel{\text { def }}{=} \mathcal{R}_{2}(r(z)) .
$$

Furthermore, $\mathcal{R}_{2}(a)>-1$ and $r\left(\mathcal{R}_{2}(a)\right)<1 / \beta$. Next, if $(a, \theta) \in \mathcal{S}$, then

$$
\gamma(a, \theta)=a^{2} \alpha(a, \theta) \frac{1-\theta+\ln \theta}{2-\theta+\ln \theta}<1 .
$$


We are now in a position to prove Theorem 2.1 for $(a, \theta) \in \mathcal{D}$. The arguments are taken from the corresponding proof of Theorem 1.1 in [13]. We include the details for the sake of completeness.

Lemma 4.13. Let $x$ be a solution of Eq. (4.9) and set $M=\limsup _{t \rightarrow \infty} x(t), m=$ $\liminf _{t \rightarrow \infty} x(t)$. If $(a, \theta) \in \mathcal{D}$, then $m=M=\lim _{t \rightarrow \infty} x(t)=0$.

Proof. We will reach a contradiction if we assume that $m<0<M$ (note that the cases $M \leq 0$ and $m \geq 0$ were already considered in Lemma 4.2).

First suppose that $(a, \theta) \in \mathcal{S}$. By Lemmas 4.9 and 4.12 we obtain that

$$
m \geq F_{1}(M)>\mathcal{R}_{2}(r(M))>-1 .
$$

Now take an arbitrary $z \geq 0$. Since $r(z) \in(a, 0]$ and $\mathcal{R}_{2}(z)$ is increasing on $(a, 0]$, we get $r\left(\mathcal{R}_{2}(r(z))\right)<1 / \beta$ from Lemma 4.12 Therefore, the rational function $\lambda \stackrel{\text { def }}{=} \mathcal{R} \circ r \circ \mathcal{R}_{2} \circ r:$ $[0, \infty) \rightarrow[0, \infty)$ is well defined. From Lemmas 4.8 and 4.10 we obtain

$$
M<\mathcal{R}\left(r\left(\mathcal{R}_{2}(r(M))\right)\right)=\lambda(M) .
$$

On the other hand, due to the inequality $\lambda^{\prime}(0)=\gamma(a, \theta)<1$ (see Lemma 4.12), we obtain that $\lambda(z)<z$ for all $z>0$, a contradiction.

Now let $(a, \theta) \in \mathcal{D}^{*}$; notice that $a \alpha(a, \theta) \in(-1,0)$ in view of (4.11). We define the rational function

$$
R:\left((a \beta-1)^{-1},+\infty\right) \rightarrow\left(-a \alpha(a \beta-1)^{-1},+\infty\right) \subset\left((a \beta-1)^{-1},+\infty\right)
$$

as $R=\mathcal{R} \circ r$. By (4.23), we have $R^{\prime}(0)=a \alpha(a, \theta) \in(-1,0)$. Next,

$$
m>R(M)>\mathcal{R}(a)>-1 .
$$

Indeed, if $M \leq a(\theta-1) / \theta-1$, then Lemmas 4.8 and 4.10 imply that $m \geq F(M)>$ $\mathcal{R}(r(M))=R(M)$. Now if $M \geq a(\theta-1) / \theta-1$, then Lemmas 4.9 and 4.11 give that $m \geq F_{1}(M)>\mathcal{R}(r(M))=R(M)$. The last inequality in (4.26) follows from Lemma 4.10

Finally, applying Lemmas 4.8 and 4.10 and (4.26), and using the inequality $R \circ R(x)<$ $x, x>0$, which holds since $(R \circ R)^{\prime}(0)=\left(R^{\prime}(0)\right)^{2}<1$, we obtain

$$
M \leq F(m)<\mathcal{R}(r(m))<\mathcal{R}(r(R(M)))=R(R(M))<M,
$$

a contradiction.

REMARK 4.14. Theorem 2.1 follows from Lemmas 4.7 and 4.13 if $b>0$ in (D1) and (D2).

For $b=0$, the same arguments work taking $r(x)=a x$. Since $r$ is linear, all involved functions are much simpler; for example, in Lemma4.7 $\chi(x)=a^{2}(1-\theta) x /(a-\theta)$ is linear. In the same way, functions $F$ and $F_{1}$ introduced in Subsection 4.4 are now linear. Indeed, we have $F(x)=\left[a^{2}+a(1-a) \exp \left(a^{-1} e^{-h}\right)\right] x$ and $F_{1}(x)=a^{2}\left[1-\exp \left(e^{-h}+h-1\right)\right] x$. We can proceed analogously to the proof of Lemma4.13, taking into account that $F^{\prime}(0)>-1$ for $(a, \theta) \in \mathcal{D}$ and $F_{1}^{\prime}(0)>-1$ for $(a, \theta) \in \mathcal{S}$. Obviously, functions $\mathcal{R}$ and $\mathcal{R}_{2}$ are not necessary in this case. 
Acknowledgments. This research was supported by FONDECYT (Chile), project 8990013. E. Liz was supported in part by MCT (Spain) and FEDER under project BFM2001-3884-C02-02. V. Tkachenko was supported in part by F.F.D. of Ukraine, project 01.07/00109. M. Pinto and S. Trofimchuk were supported by FONDECYT (Chile), projects 1030535 and 1030992, respectively.

\section{REFERENCES}

[1] K. Cooke, P. van den Driessche and X. Zou, Interaction of maturation delay and nonlinear birth in population and epidemic models, J. Math. Biol. 39 (1999), 332-352. MR1727839|(2001b:92040)

[2] K. Gopalsamy, "Stability and oscillations in delay differential equations of population dynamics," Mathematics and its Applications, 74, Kluwer, Dordrecht, 1992. MR1163190 (93c:34150)

[3] K. Gopalsamy, S.I. Trofimchuk and N.R. Bantsur, A note on global attractivity in modems of hematopoiesis, Ukrain. Math. J. 50 (1998), 5-12. MR.1669243 (2000e:92009)

[4] K. Gopalsamy and P.-X. Weng, Global attractivity and level crossings in a model of haematopoiesis, Bull. Inst. Math. Acad. Sinica 22 (1994), 341-360. MF.1311545 (96a:92004)

[5] I. Györi and G. Ladas, Oscillation Theory of Delay Differential Equations with Applications, Oxford Univ. Press, London, 1991. MR1168471 (93m:34109)

[6] I. Győri and S. Trofimchuk, Global attractivity in $x^{\prime}(t)=-\delta x(t)+p f(x(t-\tau))$. Dynam. Systems Appl. 8, (1999), 197-210. MR1695779 (2000d:34160)

[7] J. K. Hale and S. M. Verduyn Lunel, Introduction to Functional Differential Equations, SpringerVerlag, New-York, 1993. MR 1243878 (94m:34169)

[8] A. Ivanov, E. Liz and S. Trofimchuk, Halanay inequality, Yorke 3/2 stability criterion, and differential equations with maxima, Tohoku Math. J. 54 (2002), 277-295. MR1904953 (2003j:34139)

[9] Y. Kuang, "Delay differential equations with applications in population dynamics," Academic Press, 1993. MR,1218880 (94f:34001)

[10] M.R.S. Kulenovic, G. Ladas and Y.G. Sficas, Global attractivity in population dynamics, Comput. Math. Appl. 18 (1989), 925-928. MR1021318 (91c:92024)

[11] J.W. Li and S.S. Cheng, Global attractivity in an RBC survival model of Wazewska and Lasota, Quart. Appl. Math. 60 (2002), 477-483. MF1914437 (2003f:92037)

[12] E. Liz, C. Martínez and S. Trofimchuk, Attractivity properties of infinite delay Mackey-Glass type equations, Differential and Integral Equations, 15 (2002), 875-896. MR.1895571 (2003f:34141)

[13] E. Liz, V. Tkachenko and S. Trofimchuk, A global stability criterion for scalar functional differential equations, SIAM J. Math. Anal., 35 (2003), 596-622. MR.2048402 (2004m:34175)

[14] E. Liz, M. Pinto, G. Robledo, V. Tkachenko and S. Trofimchuk, Wright type delay differential equations with negative Schwarzian, Discrete Contin. Dynam. Systems, 9 (2003), 309-321. MR1952376 (2004a:34135)

[15] M.C. Mackey and L. Glass, Oscillation and chaos in physiological control systems, Science 197 (1977), 287-289.

[16] X. H. Tang and X. Zou, Stability of scalar delay differential equations with dominant delayed terms, Proc. Roy. Soc. Edinburgh Sect. A, 133 (2003), 951-968. MR2006211 (2004h:34148) 